\title{
Robust feeder reconfiguration in radial distribution networks
}

\author{
Carlos Henrique Nogueira de Resende Barbosa ${ }^{\mathrm{a}, \mathrm{c}, *}$, Marcus Henrique Soares Mendes ${ }^{\mathrm{b}, \mathrm{c}}$, \\ João Antônio de Vasconcelos ${ }^{c}$
}

\author{
${ }^{a}$ Federal University of Ouro Preto, Department of Electrical Engineering, Rua 37, no. 115, Loanda, CEP: 35931-026 João Monlevade, MG, Brazil \\ ${ }^{\mathrm{b}}$ Federal University of Viçosa, Campus Florestal, Rodovia LMG 818, km 6, CEP: 35690-000 Florestal, MG, Brazil \\ ${ }^{\mathrm{c}}$ Federal University of Minas Gerais, Graduate Program in Electrical Engineering, Av. Antônio Carlos, 6627 - Pampulha, CEP: $31270-901$ Belo Horizonte, MG, Brazil
}

\section{A R T I C L E I N F O}

Article history:

Received 21 May 2012

Received in revised form 25 July 2013

Accepted 21 August 2013

\section{Keywords:}

Interval analysis

Feeder reconfiguration

Multi-objective optimization

Radial distribution networks

\begin{abstract}
A B S T R A C T
Distribution feeder reconfiguration has been an active field of research for many years. Some recent theoretical studies have highlighted the importance of smart reconfiguration for the operating conditions of such radial networks. In general, this problem has been tackled using a multi-objective formulation with simplified assumptions, in which the uncertainties related to network components have been neglected by both mathematical models and solution techniques. These simplifications guide searches to apparent optima that may not perform optimally under realistic conditions. To circumvent this problem, we propose a method capable of performing interval computations and consider seasonal variability in load demands to identify robust configurations, which are those that have the best performance in the worst case scenario. Our proposal, named the Interval Multi-objective Evolutionary Algorithm for Distribution Feeder Reconfiguration (IMOEA-DFR), uses interval analysis to perform configuration assessment by considering the uncertainties in the power demanded by customers. Simulations performed in three cases on a 70-busbar system demonstrated the effectiveness of the IMOEA-DFR, which obtained robust configurations that are capable to keep such system working under significant load variations. Moreover, our approach achieved stable configurations that remained feasible over long periods of time not requiring additional reconfigurations. Our results reinforce the need to include load uncertainties when analyzing DFR under realistic conditions.
\end{abstract}

(c) 2013 Elsevier Ltd. All rights reserved.

\section{Introduction}

Reconfiguration of radial distribution networks is a viable option for ensuring optimal or nearly optimal operation in the presence of several constraints and variable power demand $[7,16,18$ $22,35]$. From a theoretical perspective, distribution networks can always be reconfigured whenever imminent loading variations are detected. Even though optimization procedures may reveal improvements on power losses, voltage flatness or load balancing related to a given distribution network, it is important to realize that maneuvers performed by power utilities are still costly, lack appropriate equipment [35], and hence, they are unusual when such systems are operating normally. Although feeder reconfiguration seems to be very attractive to optimize the system's operating conditions, supervisory centers adopt a conservative strategy to avoid switching maneuvers as much as possible. This behavior can be justified by the low level of network automation and the

\footnotetext{
* Corresponding author at: Federal University of Ouro Preto, Department of Electrical Engineering, Rua 37, no. 115, Loanda, CEP: 35931-026 João Monlevade, MG, Brazil. Tel.: +55 31 34093426; fax: +55 3134095480 .

E-mail addresses: carloshnrb@gmail.com C.H.N. de Resende Barbosa), marcus. mendes@ufv.br (M.H.S. Mendes), jvasconcelos@ufmg.br (J.A. de Vasconcelos).
}

imminent risks due to interference with protective devices, which may cause unexpected overloads or interruptions in the power supply. From the practical standpoint, maneuvers are the preferred procedure for solving critical or abnormal situations, but rarely for performance optimization.

Under normal conditions, seasonal variations in power demand require utility companies to rely on demand estimation models to properly meet demand within safety margins and quality indexes $[4,6,16]$. In these models, consumers are statistically classified according to their average demand [6]. Loading forecasts contain inherent errors because these approaches are based entirely on estimations. In addition, renewable energy has given its contribution to the power demand variation on load buses which originally acted as strict consumers. Its importance to the distribution systems may no longer to be neglected once the growing proportion of power generated by alternative means is intended to fill the gap between peak load demands and energy availability at regional extent [7].

A naive approach would treat network parameters as exact or definite values, and this approach could mistakenly assess a given configuration as optimal, adequate or fair to uncertainty-free environments. However, these configurations may be insufficient or even impracticable in the presence of power demand uncertainty. 


\section{Nomenclature}

$N_{B} \quad$ bus set size

$N_{L} \quad$ distribution line set size

$N_{s} \quad$ number of sources

$N_{w} \quad$ total number of switches

$N_{f} \quad$ number of objective functions

$N_{v} \quad$ number of uncertainty parameters

$\boldsymbol{x} \quad$ binary vector containing all the switches statuses

$\boldsymbol{x}^{c} \quad$ cth configuration

$\boldsymbol{B}^{s} \quad$ set of all buses supplied by source $s$

$Z_{i} \quad$ impedance of the $i$ th distribution line

$I_{i} \quad$ current flow in the $i$ th distribution line

$I_{i}^{\max }$

$V_{k}$

$V_{k}^{\text {nom }}$ ampacity (maximum current) of the $i$ th distribution line actual voltage at bus $k$

nominal or normalized (p.u.) voltage at bus $k$
$\|X\| \quad$ size of a given set $\boldsymbol{X}$

$|v| \quad$ modulus of the variable $v$

$\mathfrak{R}$ set of real intervals

[a] interval number

[a] interval vector

$\mathbf{a}^{+} \quad$ upper bound of an interval vector

P set of uncertainties

$\boldsymbol{X}$ set of decision variables

$\boldsymbol{p} \quad$ vector of uncertainty parameters

$\boldsymbol{f} \quad$ vector of objective functions

g vector of inequality constraints

$\boldsymbol{h}$ vector of equality constraints
Hence, it is important to obtain robust solutions. In our approach, robust solutions are those solutions that are less susceptible to uncertainties and they are able to maintain a system normally operating even in the worst case scenario. In this work, we restricted uncertainties to PQ loads, because they are among the most relevant factors in feeder reconfiguration decisions, mainly if we consider power generation in those buses [12,32-34]. Because feeder reconfiguration depends on some power flow method [2], uncertainties must be accounted for using a mathematical model capable of reflecting all immediate effects on the power flow $[11,12,32,33]$.

Feeder reconfiguration is considered a combinatorial problem with an irregular search space [26,31] and is generally formulated as a restricted multi-objective problem. The radiality constraint and the discrete nature of the switches prevent the use of classical techniques to solve feeder reconfiguration $[2,18,19,23,25,27]$. An evolutionary stochastic approach was chosen because this approach is relatively unaffected by the nature of the problem. As previously mentioned, an efficient solution set in a deterministic environment can be partially or totally impaired in a perturbed environment, which moves these solutions away from the Pareto frontier [14]. Thus, a conventional approach will probably fail to identify an efficient solution set to the feeder reconfiguration problem. Therefore, we present a proper implementation, in which a Multi-Objective Evolutionary Algorithm (MOEA) based on NSGAII [13] was coupled to an interval version of the Backward/Forward Sweep Method (BFSM). This novel hybrid approach, which we call Interval Multi-objective Evolutionary Algorithm for Distribution Feeder Reconfiguration (IMOEA-DFR), is a robust method of solving reconfiguration problems. To our knowledge, there is no study encompassing this specific issue yet. The objectives of this work are (i) to provide a new strategy that properly addresses load uncertainties in DFR optimization (see Section 5), (ii) to demonstrate the fragility of optimal solutions found by conventional approaches in uncertain real-world environments (see case 2 in Section 6), and (iii) to discuss how robust solutions can replace pseudo-optimal solutions to ensure reasonable and satisfactory network operating conditions in the worst case scenario (see case 3 in Section 6).

This paper is organized as follows. In Section 2, related works are cited, and their primary contributions are highlighted. The formulation of the DFR problem is presented in Section 3. In Section 4, the background of interval analysis is addressed, and the robust version of the DFR problem is defined. The implemented algorithm and related issues are described in Section 5. Finally, a discussion about computational experiments is presented in Section 6, and concluding remarks are given in Section 7.

\section{Related works}

The feeder reconfiguration has been treated as a typical uncertainty-free problem in many papers [2,3,7-10,15,25,26,29]. From a classical perspective, these approaches are satisfactory, and their solution sets are valid whenever uncertainties are negligible. However, these solutions are not as reliable as they seem [5]. It has been shown that electrical power systems are subjected to many sources of uncertainty such as power (load) demand [16,20-22], distributed generation $[21,22]$, and electrical parameters [5,6,35].
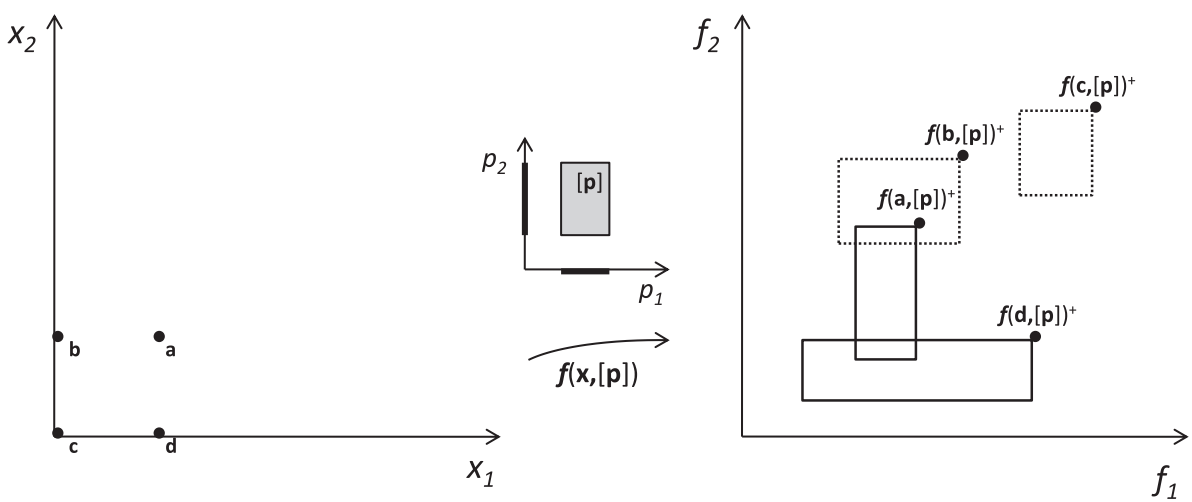

Fig. 1. Computation of the worst case performance for some solutions in the decision variable space - 2D example. 
(a)

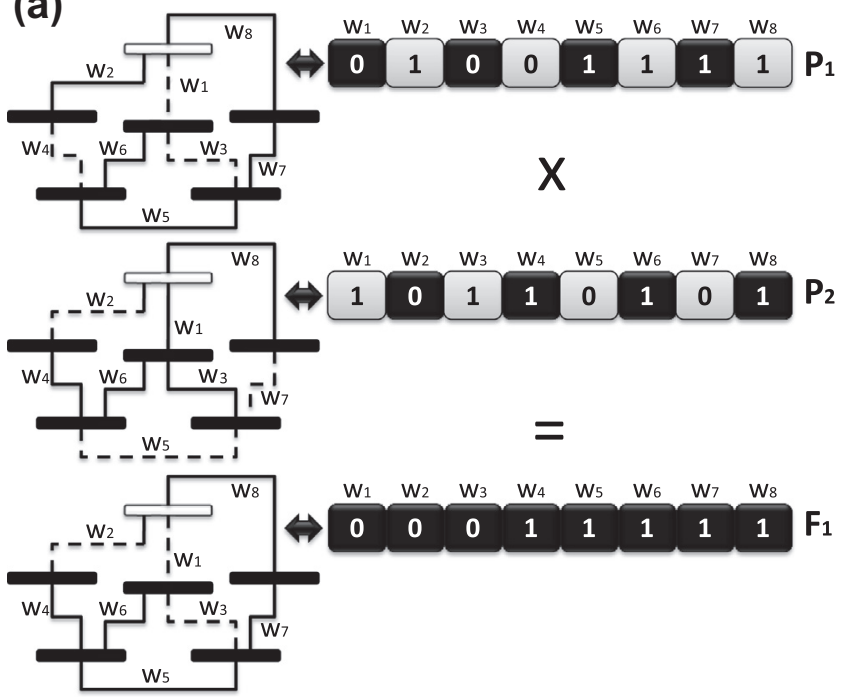

(b)

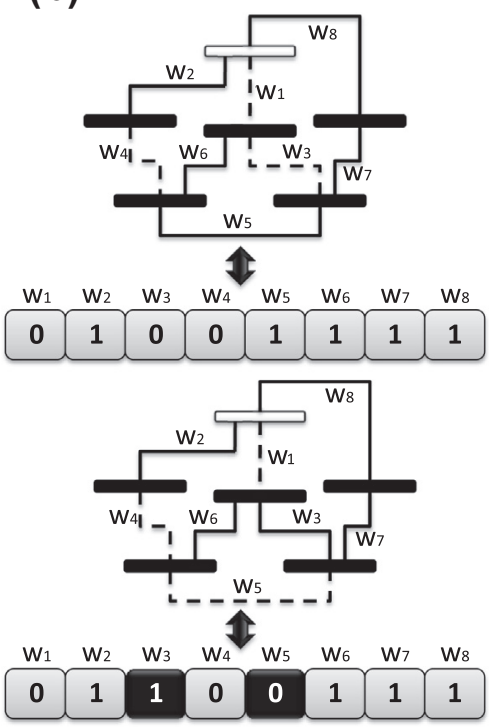

(c)

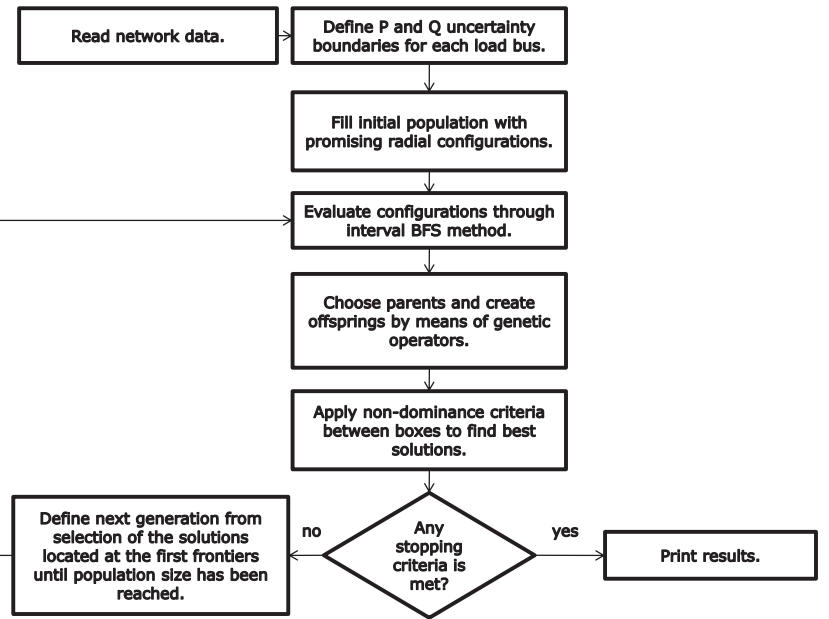

Fig. 2. (a) Crossover operator, (b) mutation operator, and (c) IMOEA-DFR flowchart.

Due to the importance of these uncertainties regardless their nature in the reconfiguration problem, seen in Section 1, it is noteworthy to mention the works that have addressed power flow methods under uncertain environments. Some authors have proposed versions of power flow analysis to cope with uncertainties $[6,12,32,34,35]$. Load variations were modeled for network planning purposes by Chaturvedi et al. [6] and later by Borges and Martins [4]. In [6], a proposal was devised based on interval arithmetic approach that represents loads through Gaussian probability density functions. Vaccaro presented an alternative to the samplingbased technique applied to radial power flow analysis supported by interval mathematics [32] or affine arithmetic [33]. He was the first to introduce an affine arithmetic approach [33] to find the distribution network's operating point, while considering variation in load and generation levels. His proposals were intended to tighten variables' domains without losing valid solutions. Still, fewer loadflow methods are able to deal with uncertainties in radial networks $[4,6,11,27]$. Das used an interval analysis technique to perform power flow analysis in unbalanced [12] and balanced [11] three-phase radial distribution systems while considering load and feeder parameters uncertainties. Zhang et al. [35] proposed a method called Reliability-Oriented Reconfiguration (ROR) which employed interval analysis to quantify the impact of uncertain data on system parameters and to maximize the possi- bility of reliability improvement and/or loss reduction achieved through reconfiguration.

As previously detailed, many researchers have studied strategies for coping with uncertainties in power flow analysis to tackle reconfiguration problem in a broader manner. This work goes farther and deals suitably with the robust DFR problem focusing on network operation. Our IMOEA-DFR approach applies interval analysis not only to power flow algorithms, but also to non-dominance assessments among solutions in terms of the worst case performance. Therefore, the primary contribution of this manuscript is to provide an effective method of finding robust configurations that guarantee the operating conditions of distribution feeders under uncertain loading.

\section{Problem formulation}

Our mathematical formulation is derived from [3]. The on/off statuses of all $N_{w}$ maneuverable switches were used as optimization variables and coded into a binary vector $\boldsymbol{x}\left(\boldsymbol{x}=\left[x_{1}, \ldots, x_{i}, \ldots\right.\right.$, $\left.x_{N w}\right]$ ) whose $i$ th element indicates whether the corresponding switch is open $\left(x_{i}=0\right)$ or closed $\left(x_{i}=1\right)$. Each distribution line has its own switch $\left(N_{L}=N_{w}=\|\boldsymbol{x}\|\right)$. As in [15], four objectives were assumed in this formulation: Power Losses (PL), Current Loading 


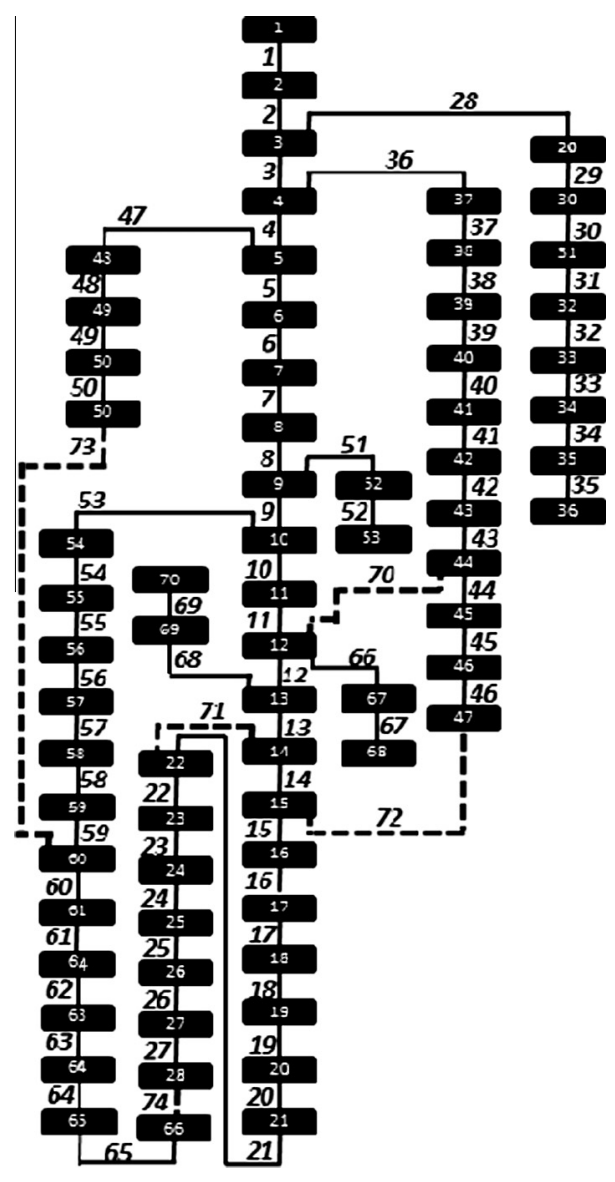

Fig. 3. Test feeder with 70 busbar found in $[2,9,15]$.

Index (CLI), Voltage Deviation Index (VDI), and Number of Switching Maneuver (NS). PL can be described as follows:

$\mathrm{PL}=\left(\sum_{i=1}^{N_{w}} x_{i} * \operatorname{Re}\left(Z_{i}\right) *\left|I_{i}\right|^{2}\right)$,

where $\operatorname{Re}\left(Z_{i}\right)$ is the real component of the $i$ th line impedance and $\left|I_{i}\right|$ corresponds to the magnitude of the current flowing through the $i$ th line. The second objective is mathematically represented as:

$\mathrm{CLI}=\left(\frac{1}{N_{w}} \sum_{i=1}^{N_{w}} x_{i} * I_{i} / I_{i}^{\max }\right)$.

The third objective involves minimal voltage deviation from the nominal values at all $N_{B}$ buses in the system. To implement this criterion, the index proposed by Sahoo et al. [25], which requires simple computations, was modified by Huang and represented as [15]:

$\mathrm{VDI}=\left(\frac{1}{N_{B}} \sum_{k=1}^{N_{B}} x_{i, k}^{C} *\left|V_{k}-V_{K}^{\text {nom }}\right|\right), \quad x_{i, k}^{C} \in \boldsymbol{W}$,

where $\boldsymbol{W}$ denotes a set of all closed switches and $x_{i, k}^{c}$ indicates that switch $i$ is closed to connect bus $k$ to a power node in configuration $c$. The fourth objective considers the number of switching maneuvers, which must be kept as small as possible due to operational costs and network management considerations. This objective is calculated using the Manhattan distance:

$\mathrm{NS}=\sum_{i=1}^{N_{w}}\left|x_{i}^{c}-x_{i}^{0}\right|$ where $x_{i}^{c}$ and $x_{i}^{0}$ denotes each set of corresponding switches in the candidate and previous configurations, respectively. The distance between any corresponding switch equals $\left|x_{i}^{c}-x_{i}^{0}\right|$. Additional, restrictions were admitted. Radiality must be ensured using the following relations:

$$
\begin{aligned}
& N_{B}-N_{s}=\|\boldsymbol{W}\|=\sum_{i=1}^{N_{W}} x_{i}^{c}, \\
& \sum_{s=1}^{N_{s}} b_{k}^{s}=1, \quad \forall k=1, \ldots, N_{B}, \\
& x_{i, k}^{c} \leqslant x_{i-1, k-1}^{c} \mid x_{i-1, k-1}^{c}=1 \Longleftrightarrow B^{s}:=B^{s} \cup b_{k-1}^{s} \\
& \quad \forall i, \quad i-1=2, \ldots, N_{w}, \quad \forall k=2, \ldots, N_{B} .
\end{aligned}
$$

The condition in Eq. (5.a) holds for any $c$ radial configuration or set of configurations, but it does not guarantee radiality by itself. In Eq. (5.b), $b_{k}^{s}$ is a binary variable that takes the value one (zero) to indicate that bus $k$ is fed (not fed) by source $s$. This statement is complemented by Eq. (5.c), wherein the status of the $i$ th switch $\left(x_{i, k}^{c}\right)$, which delivers power to the $k$ th bus, depends on the status of an adjacent switch, $\left(x_{i-1, k-1}^{c}\right)$, closer to the (temporary) common source $s$, which in turn delivers power to the $k-1$ th bus. All of the $N_{B}$ buses must be connected to available sources by a unique path to avoid loop or islanding. The following restriction ensures that the current in each line (or branch) does not exceed its ampacity:

$\left|I_{i}\right| \leqslant I_{i}^{\max }, \quad i \in \boldsymbol{W}$.

The final restriction establishes that no valid solution may violate upper and lower limits of the bus voltages:

$V_{k}^{\min } \leqslant V_{k} \leqslant V_{k}^{\max }, \quad k=1, \ldots, N_{B}$.

The values of $V_{k}^{\min }$ and $V_{k}^{\max }$ were assumed to be 0.9 p.u. and 1.05 p.u. on our simulations, respectively, according to the values defined in Baran's work [2]. It is worthy to note that voltage restriction is complementary to Eq. (3) whose goal is to reduce as much as possible bus voltage variation.

\subsection{Component modeling issues}

Power can be re-routed through reconfiguration, i.e., opening and closing sectionalizing and tie switches. Reconfiguration is an effective way to operate distribution networks under load demands changes by redistributing currents through various lines to prevent or alleviate overloading in system equipment, reduce real power losses, improve the voltage profile and increase system stability. Feeders may serve a variety of different consumers who can be organized into classes such as residential, commercial, or industrial [16]. Each class has its own peak time, and their load behaviors affect the overall loading over the 24-h cycle. Depending on the chosen configuration and distribution of those classes, restrictions can be violated in some instances. In these cases, the system must be reconfigured in advance to avoid potential risks to system stability (e.g., equipment overloading or non-prescribed bus voltages).

In one of the experiments described in Section 6, loads are modeled as power sinks with variations prescribed by demand curves [28]. Seasonal behavior is incorporated into the loads according to the five profiles found in [28]. These profiles were assigned to various loads according to their nominal values, which are specified in the original test feeder. Moreover, system parameters (e.g., line impedance and available power) are treated as discrete, error-free quantities. Each demand curve statistically describes a group of similar consumers with identical profiles and thus has 

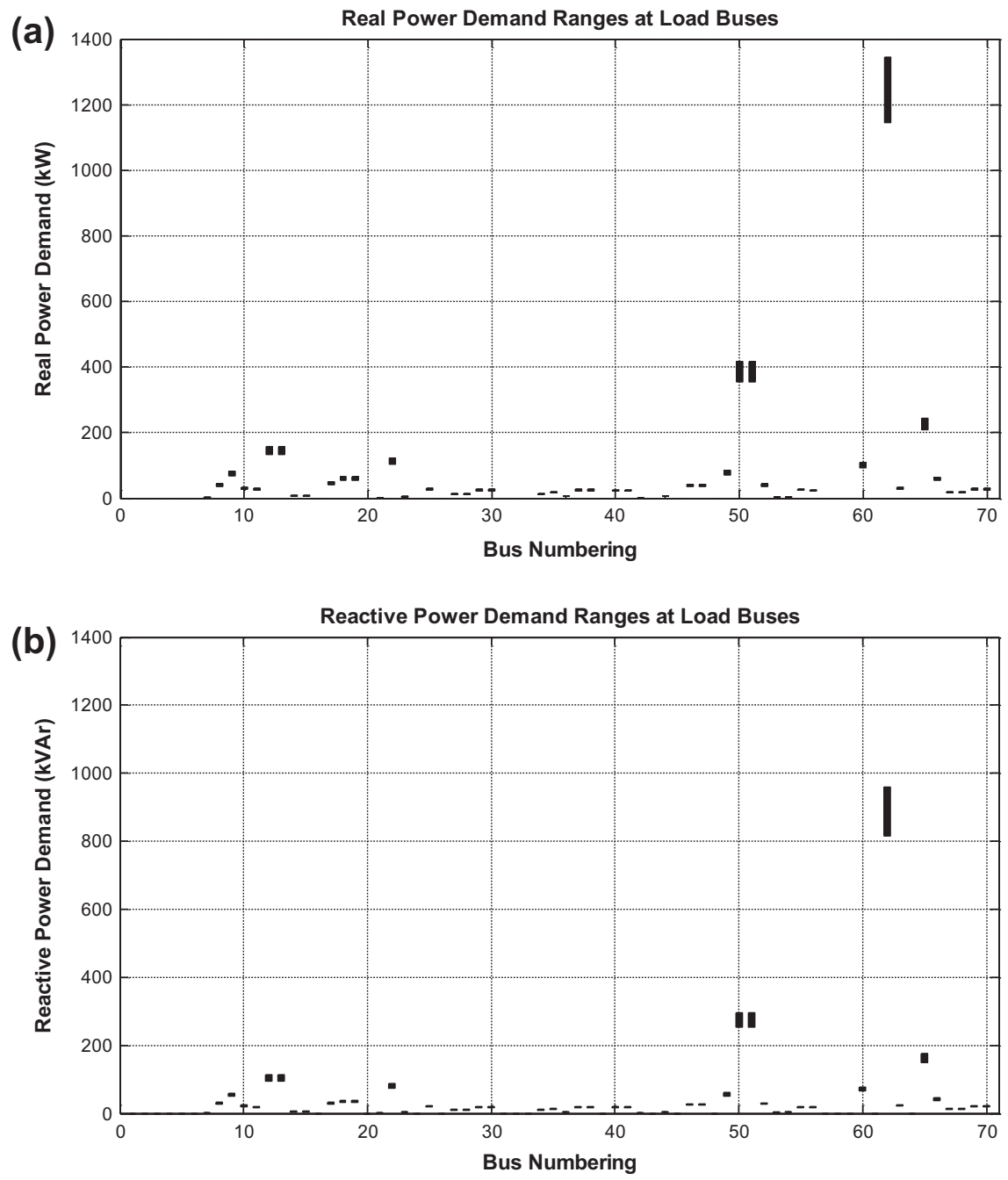

Fig. 4. Ranges of the (a) real and (b) reactive power demands of the load buses in case 1 .

an inherent degree of uncertainty. We incorporate uncertainties by assigning specified ranges to each bus. Loads can vary considerably throughout the day. Thus, the current configuration may be suboptimal or even invalid due to violations of some restrictions. These variations can be understood as perturbations to the system. In this work, a perturbation is characterized by well-defined uncertainty ranges on specific buses. To account for uncertainties, real and reactive power loads at the buses were treated as interval numbers.

\section{Interval analysis}

This section addresses the basic concepts and definitions of Interval Analysis (IA) required to understand how the proposed algorithm deals with intervals. More detailed information can be found in [17]. IA approaches use a range-based model that represents a punctual number and its uncertainty. The model presented in this work uses interval numbers to indicate the spread of a numerical value. A real, closed and connected interval is defined as:

Definition 1. A closed interval [a] is a connected subset in $\mathfrak{R}$ defined by

$[a]=\left[a^{-}, a^{+}\right]:=\left\{a \in \mathfrak{R} \mid a^{-} \leqslant a \leqslant a^{+}\right\}$, where $a^{-}$and $a^{+}$represent the lower and upper bounds of $[a]$, respectively. The set of all intervals defined by (8) represents the set of real intervals $(\mathfrak{R})$.

Definition 2. The multi-dimensional real interval $[\mathbf{a}] \in \mathfrak{R}^{n}$, denominated box or interval vector is given by

$[\mathbf{a}]=\left[a_{1}\right] \times\left[a_{2}\right] \times \ldots \times\left[a_{n}\right]$.

Definition 3. The lower bound $\mathbf{a}^{-}$of $[\mathbf{a}]$ is given by $\mathbf{a}^{-}=\left(a_{1}^{-}, a_{2}^{-}, \ldots, a_{n}\right)^{T}$. In the same way, the upper bound $\mathbf{a}^{+}=\left(a_{1}^{+}, a_{2}^{+}, \ldots a_{n}^{+}\right)^{T}$.

Definition 4. The four classical operations of real arithmetic can be extended to intervals. For any such binary operator, denoted by $\Theta$, perform the operation means computing

$[a] \Theta[b]:=\{a \Theta b \mid a \in[a] \wedge b \in[b]\}$.

Regarding the extreme bounds, each classical operation for nonempty closed interval is given by:

$[a]+[b]=\left[a^{-}+b^{-}, a^{+}+b^{+}\right]$

$[a]-[b]=\left[a^{-}-b^{+}, a^{+}-b^{-}\right]$. 

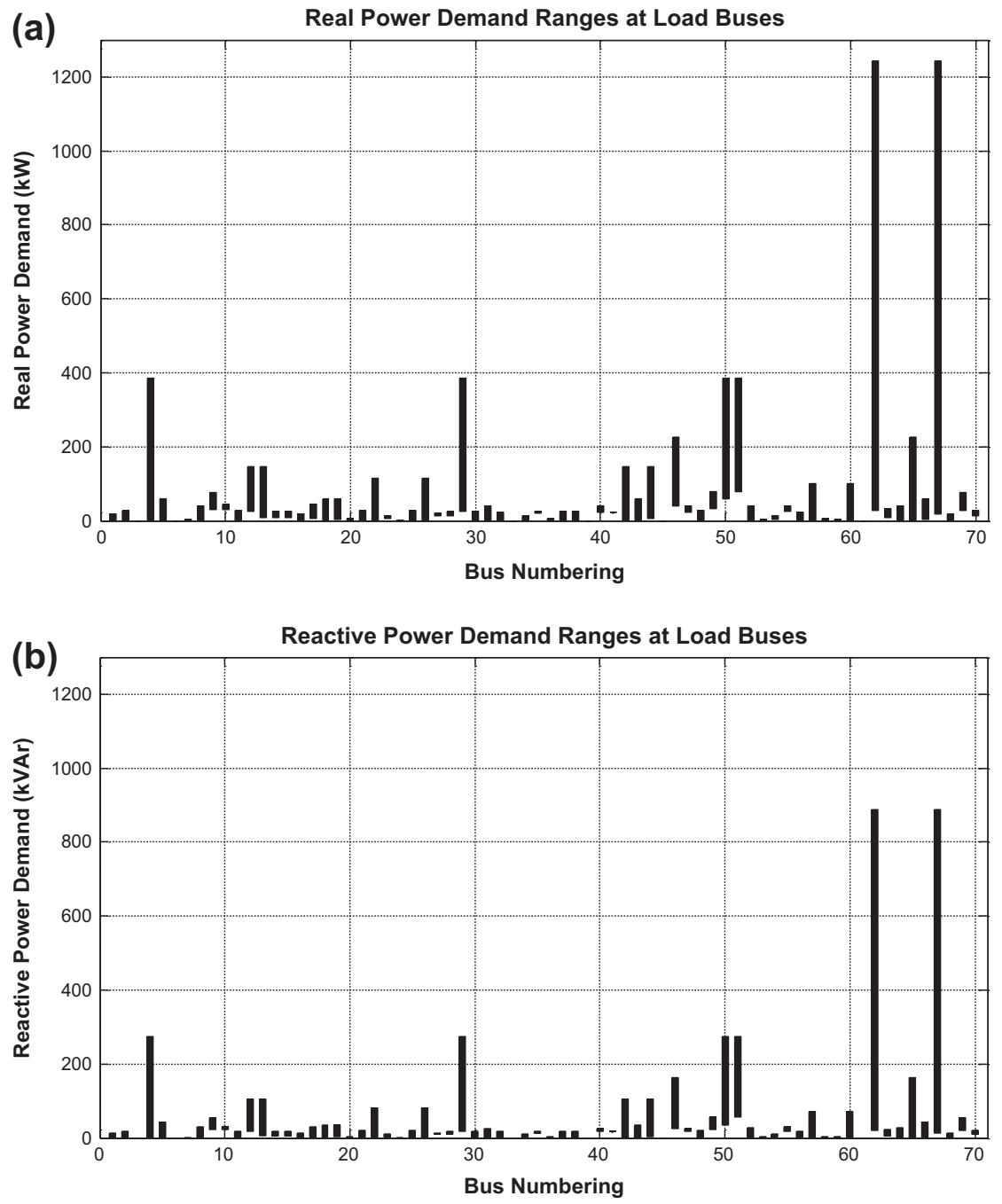

Fig. 5. Ranges of the (a) real and (b) reactive power demands of the load buses in case 2 .

$[a] *[b]=[\min (s), \max (s)]$, being

$s=\left\{a^{-} * b^{-}, a^{-} * b^{+}, a^{+} * b^{-}, a^{+} * b^{+}\right\}$

$[a] /[b]=[a] *(1 /[b])$,

Where

$1 /[b]=\phi$, if $[b]=[0,0]$.

$1 /[b]=\left[1 / b^{+}, 1 / b^{-}\right]$, if $0 \notin[b]$.

$1 /[b]=\left[1 / b^{+}, \infty\right]$, if $b^{-}=0 \wedge b^{+}>0$.

$1 /[b]=\left[-\infty, 1 / b^{-}\right]$, if $b^{-}<0 \wedge b^{+}=0$.

$1 /[b]=[-\infty, \infty]$, if $b^{-}<0 \wedge b^{+}>0$.

It is noteworthy that the interval addition, subtraction, multiplication, and division operators operate on any two interval operands to produce an interval result that contains all arithmetical combinations of the interval numbers describing the operands.

As shown in the next subsection, we represent the power demands on the PQ buses using interval numbers to guarantee that all uncertainties on the loads are taken into account. In terms of implementation, every real operation that uses interval variables is replaced by the respective interval operator in the power flow analysis algorithm. Therefore, the fundamental theorem of IA [17] guarantees that all obtained outputs are contained within the interval result.

IA includes an inherent pessimism related to the interval operations, meaning that the output interval may be overestimated. For instance, the real expressions $f=x *(1-x)$ and $g=x-x^{2}$ are equivalent in real arithmetic. However, assuming $[x]=[0,1]$, their interval counterparts lead to distinct results given that $f[x]=[0,1]$ and $g[x]=[-1,1]$. Both results represent an enclosure of the correct output regarding the input interval. On the other hand, the former is narrower (is less pessimistic) than the latter. In general, this pessimism arises as a result of multiple occurrences of the same variable. Mathematical reorganization of the expression can resolve this problem, but variable multiplicity may not always be avoidable.

\subsection{Non-dominance analysis in an interval context}

In this paper, we compute the worst case performance of each solution by considering the uncertainty parameters (uncertainty in the load demand on each PQ bus) using intervals. The worst case performance of a configuration is computed using:

$f_{w c}(\boldsymbol{x}, \boldsymbol{p})=\max _{\boldsymbol{p} \in \mathbf{P}} f_{i}(\boldsymbol{x}, \boldsymbol{p}), \quad i=\left\{1, \ldots, N_{f}\right\}$.

In Fig. 1, three spaces representing the uncertainty, decision variable, and objective are represented in two dimensions to illustrate the procedure by which the objective functions are evaluated for each configuration in $\boldsymbol{X}$ by considering perturbations in $\mathbf{P}$. Thus, a configuration is associated with a box in the objective space. The 

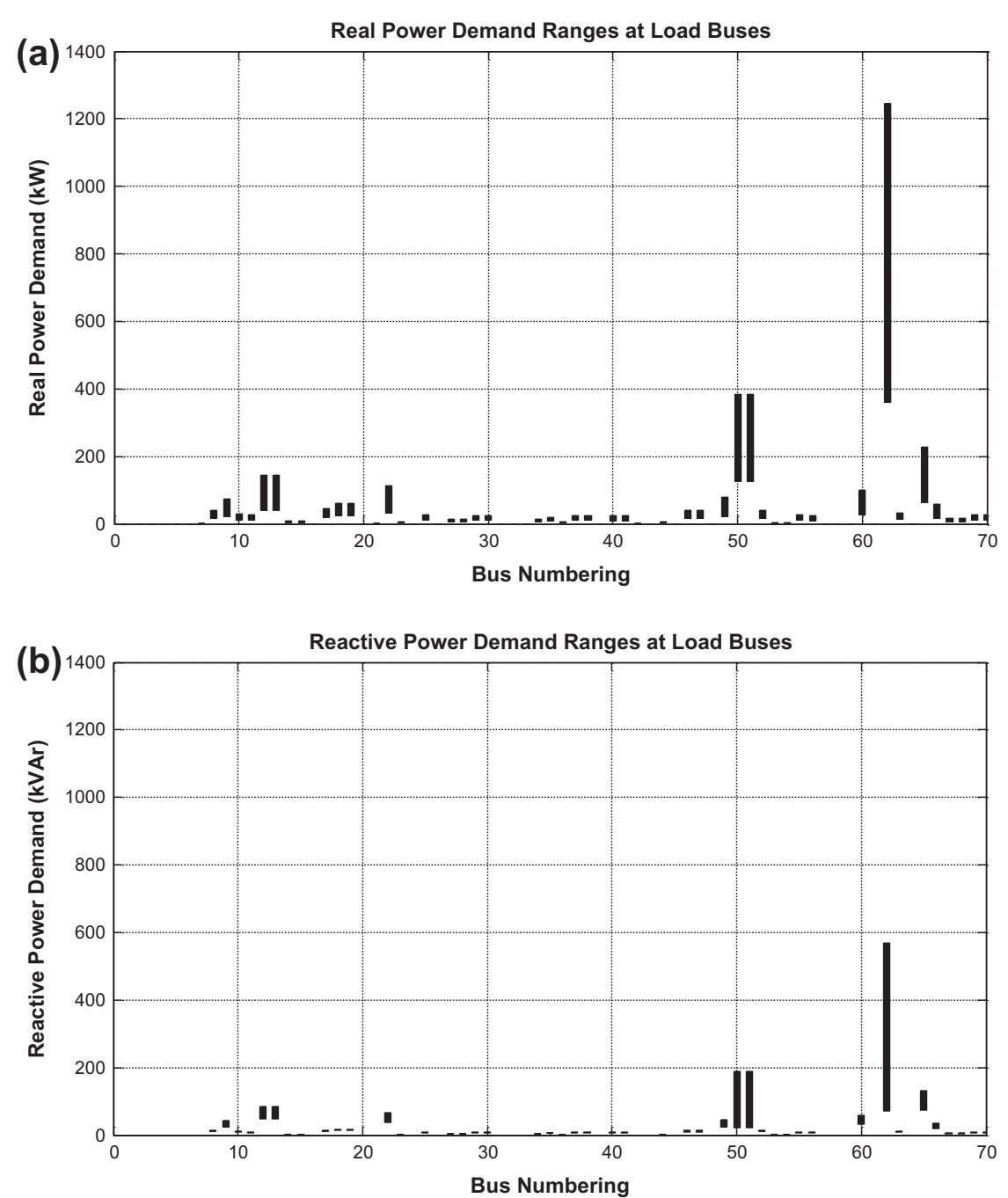

Fig. 6. Ranges of the (a) real and (b) reactive power demands of the load buses in case 3 .

Table 1

Optimal solutions to the 70-busbar system (the last two data columns consider $8 \%$ uncertainty for all loads).

\begin{tabular}{|c|c|c|c|c|}
\hline Parameter & $\begin{array}{l}\text { Reference solution found in } \\
\text { [15] }\end{array}$ & $\begin{array}{l}\text { MOEA-DFR } \\
\text { solution }\end{array}$ & $\begin{array}{l}\text { Reference solution considering uncertainty } \\
\text { effects }\end{array}$ & IMOEA-DFR solution \\
\hline Real power loss (kW) & 113.406 & 113.406 & {$[96.5678,134.125]$} & {$[96.592,134.1582]$} \\
\hline Voltage deviation index & 0.0129 & 0.0129 & {$[0.011851,0.013959]$} & $\begin{array}{l}{[0.011804} \\
0.013905]\end{array}$ \\
\hline Average current loading index & 0.1860 & 0.1860 & {$[0.17297,0.20342]$} & {$[0.17268,0.20308]$} \\
\hline Switching number & 4 & 4 & 4 & 4 \\
\hline Overall minimal bus voltage (p.u.) & 0.9288 & 0.9288 & {$[0.92288,0.93471]$} & {$[0.92288,0.93471]$} \\
\hline Maximum current loading index & 0.9203 & 0.9203 & {$[0.84664,0.99388]$} & {$[0.84664,0.99388]$} \\
\hline $\begin{array}{l}\text { Proposed solution (opened } \\
\text { switches) }\end{array}$ & $\{13,59,70,71,74\}$ & $\{13,59,70,71,74\}$ & $\{13,59,70,71,74\}$ & $\{14,59,70,71,74\}$ \\
\hline
\end{tabular}

worst case performance is represented by the black filled-in circle on the upper right corner of each box. According to Definition 6, the dotted boxes are dominated by at least one of the other boxes.

Definition 5. Given that $u$ and $\boldsymbol{v} \in \mathfrak{R}^{N f}, u=\left(u_{1}, u_{2}, \ldots, u_{N f}\right)$ dominates $v=\left(v_{1}, v_{2}, \ldots, v_{N f}\right)$, denoted by $u<v$, if $f \forall i \in\{1, \ldots$, $N f\}, u_{i} \leqslant v_{\mathrm{i}} \wedge \exists i \in\{1, \ldots, N f\} \mid u_{i}<v_{\mathrm{i}}$.

Definition 6. The dominance operator is extended to interval computation as follows

$[\mathbf{a}] \prec[\mathbf{b}] \Longleftrightarrow a^{+} \prec b^{+}$.

\subsection{The robust formulation}

The worst case Robust Multi-objective Optimization Problem (RMOP) can be seen as maximizing the effects of uncertainty on the objective functions, and subsequently minimizing all objective functions, as described in [30]. Mathematically, given that $\boldsymbol{x} \in \boldsymbol{X} \subseteq \mathfrak{R}^{N w}, \boldsymbol{p} \in \mathbf{P} \subseteq \mathfrak{R}^{N p}$ and $\boldsymbol{f}(\boldsymbol{x}, \boldsymbol{p}): \mathfrak{R}^{N w} \times \mathfrak{R}^{N p} \mapsto \mathfrak{R}^{N f}$ the RMOP is defined by:

$\min _{\mathbf{x} \in S} \max _{\boldsymbol{p} \in \mathbf{P}} \boldsymbol{f}(\boldsymbol{x}, \boldsymbol{p})=\left\{f_{1}(\boldsymbol{x}, \boldsymbol{p}), \ldots, f_{N_{f}}(\boldsymbol{x}, \boldsymbol{p})\right\}$,

where the feasible space is given by: 
Table 2

The best frontier found by MOEA-DFR or IMOEA-DFR in three scenarios: (i) original scenario described in [15], (ii) the perturbed scenario, and (iii) the interval scenario.

\begin{tabular}{llll}
\hline $\begin{array}{l}\text { Switching } \\
\text { number }\end{array}$ & $\begin{array}{l}\text { Original scenario } \\
(40 \text { solutions })\end{array}$ & $\begin{array}{l}\text { Perturbed scenario } \\
(65 \text { solutions })\end{array}$ & $\begin{array}{l}\text { Interval scenario } \\
(75 \text { solutions })\end{array}$ \\
\hline 0 & $1^{\text {a }}$ & $1^{\text {a }}$ & $1^{\text {a }}$ \\
2 & 1 & 11 & 3 \\
4 & 4 (Huang solution & $20^{\mathrm{b}}$ & $11^{\mathrm{b}}$ \\
& included) & & \\
6 & 15 & 22 & 30 \\
8 & 19 & 11 & 30 \\
\hline
\end{tabular}

a Initial solution.

b Huang solution was discarded due to dominance criteria.

$S:=\{\boldsymbol{x} \in X \mid \boldsymbol{g}(\boldsymbol{x}, \boldsymbol{p}) \leqslant \mathbf{0} \wedge \boldsymbol{h}(\boldsymbol{x}, \boldsymbol{p})=\mathbf{0} \forall \boldsymbol{p} \in \boldsymbol{P}\}$

Solving (17) allows us to find a set of robust minimizers, which can be written as:

$X^{*}:=\left\{\boldsymbol{x}^{*}: \neg \exists \boldsymbol{x} \mid \max _{\boldsymbol{p} \in \mathbf{P}} \boldsymbol{f}(\boldsymbol{x}, \boldsymbol{p}) \prec \max _{\boldsymbol{p} \in \mathbf{P}} \boldsymbol{f}\left(\boldsymbol{x}^{*}, \boldsymbol{p}\right)\right\} \forall \boldsymbol{x}^{*}, \quad \boldsymbol{x} \in \mathbf{S}$

Particularizing the RMOP to the DFR problem, we have $N f=4$ objective functions: $f_{1}=$ power losses, $f_{2}=$ current loading index, $f_{3}=$ voltage deviation index, and $f_{4}=$ number of switching maneuvers. In addition, there are three constraints: $h_{1}=$ involving radiality - formed by Eqs. 5.a, 5.b, 5.c, $g_{1}$ ensuring current values less than ampacity for each line (or branch) - Eq. (6), and $g_{2}$ which is related to the upper and lower limits of bus voltages - Eq. (7). The values of $f_{1}, f_{2}, f_{3}$, and $g_{1}, g_{2}$ are directly influenced by the uncertainties $\mathbf{p}$ existing in load demands of each PQ bus. Hence, for the first three objective functions and two inequality constraints, intervals are obtained as output of the IMOEA-DFR. Since $f_{4}$ is calculated by the number of switches to be maneuvered in order to change the initial configuration into the candidate solution, the influence of uncertainties is not straightly represented in this objective function. The constraint $h_{1}$ considers the topology of the configuration. Consequently, power demand uncertainties do not have an immediate effect over configuration topology. In practical terms, $f_{4}$ and $h_{1}$ can be represented by integers and, thus, they are handled as degenerated intervals in which lower and upper bounds assume the same value.

\section{Implemented algorithm}

As stated in Section 1, DFR is a discrete combinatorial problem, and classical techniques are thus inappropriate for solving this optimization problem $[18,19,23]$, which is reinforced by the radiality constraint. Many other techniques proposed in the literature are based on heuristic search strategies. In addition to being time-consuming, these techniques are restricted to network specificities and do not guarantee an optimal solution. Evolutionary

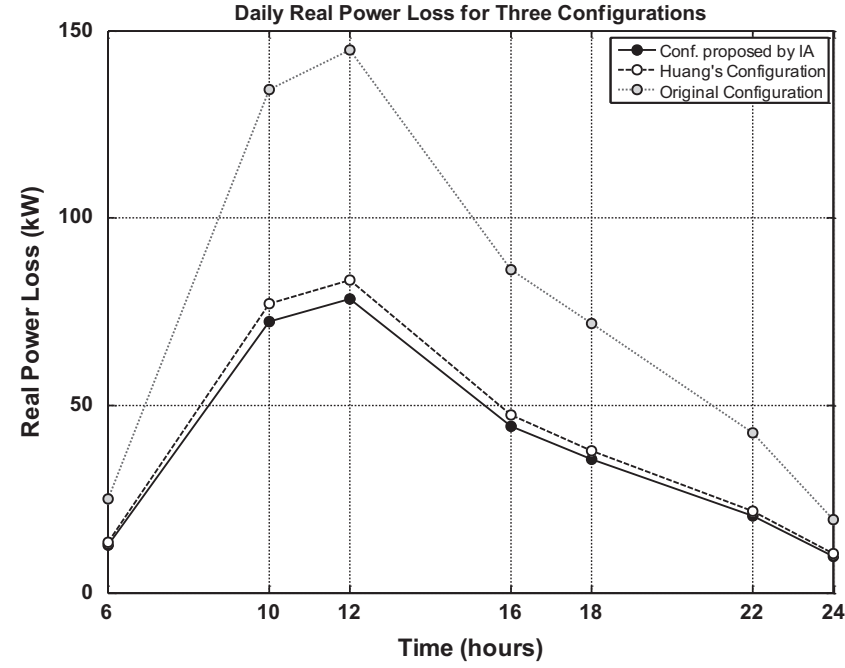

Fig. 7. Real power loss for three configurations: original, Huang and IMOEA-DFR.

algorithms are potentially capable of finding optimal or near-optimal solutions because they are not as limited as heuristics algorithms. Here, we propose an evolutionary algorithm based on NSGA-II [13] as the framework for finding non-dominated solutions. The fitness evaluation is computationally costly, and every new solution must be submitted to the iterative Backward/Forward Sweep Method (BFSM), which employs the recursive sets of equations found in [9]. Both the P-Q decoupled method and the classical Newton-Raphson method are unsuited to solving distribution network power flow. For this reason, the BFSM method with power aggregation [1] was used to compute bus voltages and power losses.

The topological analysis, which generates and modifies configurations, is another costly task and demands a considerable fraction of the overall computing time. To assure the feasibility of all evaluated individuals including those generated and those obtained from crossover and mutation operators, a Prim-like constructive heuristics is employed. A straightforward analogy can be made between the distribution system and the graph theory. Buses and distribution lines are equivalent to nodes and edges. Any distribution network can be represented as a graph $G(N, A)$ that contains a set of nodes, $N$, and edges, $A$. We employ a constructive heuristic for two purposes: (i) to create random radial configurations, and (ii) to perform crossover between two radial configurations. This method is simple and easy to implement, and solution diversity is guaranteed because every edge has the same probability of being chosen. More details about the crossover procedure based on a Prim-like heuristic are given in Algorithm 1.

Table 3

Performance comparison between Huang configuration and solution found by IMOEA-DFR. The best objective values are in italics.

\begin{tabular}{|c|c|c|c|c|c|c|c|c|c|c|}
\hline \multirow[t]{2}{*}{ Configuration } & \multicolumn{5}{|c|}{ Original scenario (nominal loads) } & \multicolumn{5}{|c|}{ Perturbed scenario (loads with uncertainty) } \\
\hline & $\begin{array}{l}\text { Real } \\
\text { power } \\
\text { loss } \\
(\mathrm{kW})\end{array}$ & $\begin{array}{l}\text { Voltage } \\
\text { deviation } \\
\text { index }\end{array}$ & $\begin{array}{l}\text { Average } \\
\text { current } \\
\text { loading } \\
\text { index }\end{array}$ & $\begin{array}{l}\text { Overall } \\
\text { minimal } \\
\text { voltage } \\
\text { (p.u.) }\end{array}$ & $\begin{array}{l}\text { Maximum } \\
\text { current } \\
\text { loading index }\end{array}$ & $\begin{array}{l}\text { Real } \\
\text { power } \\
\text { loss } \\
(\mathrm{kW})\end{array}$ & $\begin{array}{l}\text { Voltage } \\
\text { deviation } \\
\text { index }\end{array}$ & $\begin{array}{l}\text { Average } \\
\text { current } \\
\text { loading } \\
\text { index }\end{array}$ & $\begin{array}{l}\text { Overall } \\
\text { minimal } \\
\text { voltage } \\
\text { (p.u.) }\end{array}$ & $\begin{array}{l}\text { Maximum } \\
\text { current } \\
\text { loading index }\end{array}$ \\
\hline $\begin{array}{l}\text { Huang solution }[13] \\
\quad\{13,59,70,71,74\}\end{array}$ & 113.406 & 0.0129 & 0.1860 & 0.9288 & 0.9203 & 73.219 & 0.0169 & 0.1759 & 0.9582 & 0.9159 \\
\hline $\begin{array}{l}\text { Identified solution by } \\
\text { IMOEA-DFR }\{13 \\
20,59,65,70\}\end{array}$ & 107.962 & 0.0120 & 0.1872 & 0.9322 & 0.9203 & 55.902 & 0.0107 & 0.1752 & 0.9745 & 0.9159 \\
\hline
\end{tabular}




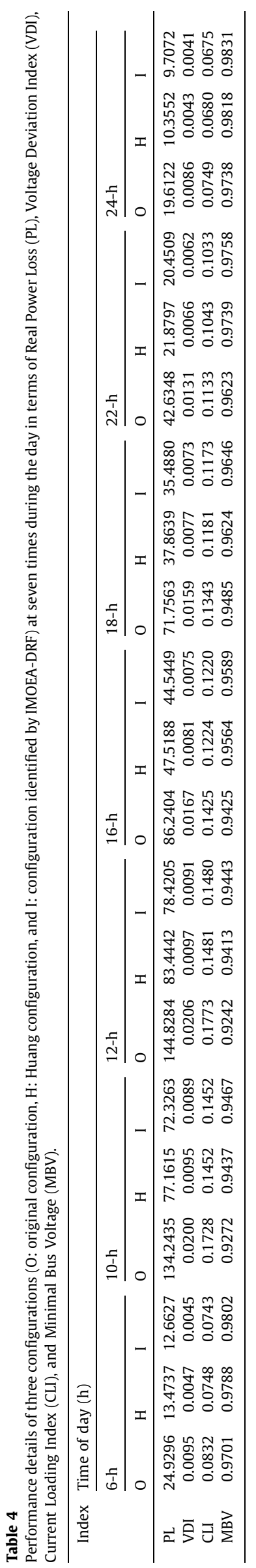

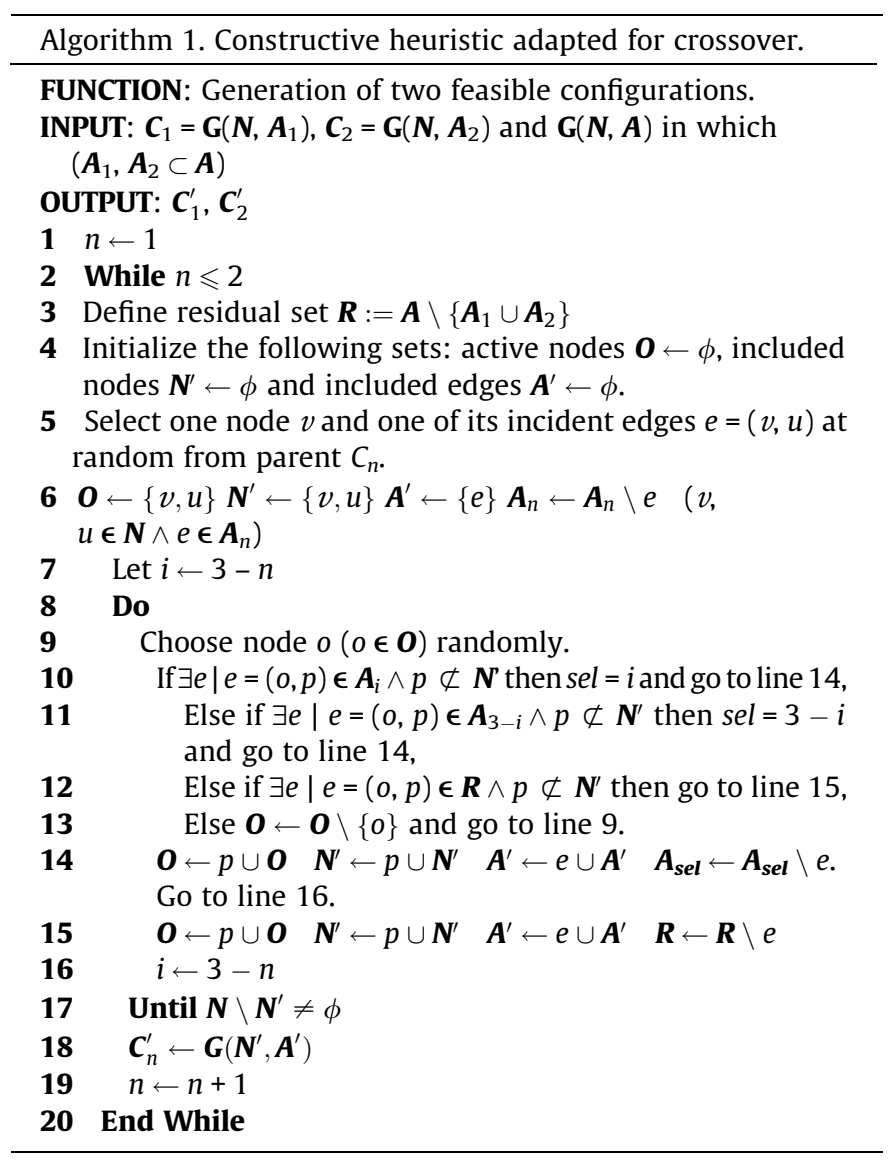

The purely constructive heuristic builds a configuration from an initial node. Subsequent nodes are added one by one, according to the available edges. If a chosen edge creates a loop, it is discarded, and it is replaced by another, chosen at random. This procedure terminates when all nodes are included. A semi-destructive heuristic is used to repair a configuration when the mutation operator is applied. This heuristic is able to detect loops as well as islanding. An open edge is closed to form a loop, and another edge belonging to this loop is opened. Although time-consuming, these two heuristics prevent useless evaluations in the proposed evolutionary algorithm and enable efficient exploration of the entire search space. Fig. 2(a and b) shows how new candidate solutions are obtained from genetic operators.

We initially implemented a non-interval algorithm, called the Multi-objective Evolutionary Algorithm for Distribution Feeder Reconfiguration (MOEA-DFR), to validate our evolutionary strategy by comparing its results with those achieved by the Enhanced Genetic Algorithm [15] (see Section 6, case 1). The MOEA-DFR is incapable of dealing with uncertainties and uses non-interval BFSM and the dominance criteria based on Definition 5 in Section 4. Next, the MOEA-DFR was improved to include the ability to deal with the worst loading uncertainty case through the use of interval computation, resulting in the IMOEA-DFR, which is illustrated in Fig. 2(c). All real mathematical operations and variables in the BFSM were replaced by their interval operator and interval variable counterparts, respectively. The algorithm only uses basic mathematical operations (addition, subtraction, multiplication and division) and two elementary functions (square root and exponentiation). In IMOEA-DFR, the non-dominance criteria are implemented according to Definition 6 in Section 4. 
Table 5

Hypervolume values for the results found by IMOEA-DFR.

\begin{tabular}{|c|c|c|c|c|c|c|c|c|c|c|c|c|}
\hline \multirow[t]{2}{*}{ Case number } & \multicolumn{10}{|c|}{ Hypervolume metric for 10 runs } & \multicolumn{2}{|c|}{ Indicators } \\
\hline & 1 & 2 & 3 & 4 & 5 & 6 & 7 & 8 & 9 & 10 & Mean & Standard deviation \\
\hline 1 & 0.0355 & 0.0349 & 0.0349 & 0.0341 & 0.0348 & 0.0357 & 0.0353 & 0.0352 & 0.0351 & 0.0349 & 0.0351 & 0.0004 \\
\hline 2 & 0.0214 & 0.0205 & 0.0212 & 0.0207 & 0.0195 & 0.0212 & 0.0212 & 0.0207 & 0.0209 & 0.0213 & 0.0208 & 0.0006 \\
\hline 3 & 0.0351 & 0.0384 & 0.0379 & 0.0377 & 0.0351 & 0.0354 & 0.0350 & 0.0352 & 0.0379 & 0.0381 & 0.0366 & 0.0015 \\
\hline
\end{tabular}

Table 6

IGD values for the results found by IMOEA-DFR.

\begin{tabular}{|c|c|c|c|c|c|c|c|c|c|c|c|c|}
\hline \multirow[t]{2}{*}{ Case number } & \multicolumn{10}{|c|}{ Inverse generational distance for 10 runs } & \multicolumn{2}{|c|}{ Indicators } \\
\hline & 1 & 2 & 3 & 4 & 5 & 6 & 7 & 8 & 9 & 10 & Mean & Standard deviation \\
\hline 1 & 0.1365 & 0.2109 & 0.3338 & 0.1518 & 0.2046 & 0.1018 & 0.2264 & 0.0526 & 0.1514 & 0.2213 & 0.1791 & 0.08 \\
\hline 2 & 0.8624 & 3.3745 & 0.5525 & 1.0353 & 1.2171 & 0.6788 & 0.7022 & 0.9399 & 0.9791 & 1.0921 & 1.1434 & 0.81 \\
\hline 3 & 0.2537 & 0.1944 & 0.2124 & 0.0414 & 0.2508 & 0.3617 & 0.3711 & 0.3884 & 0.1708 & 0.1787 & 0.2423 & 0.11 \\
\hline
\end{tabular}

\section{Experimental results}

Experiments were performed with a known feeder that has already been analyzed in the literature by Huang [15] and Chiang and Jumeau [8]. This feeder was originally presented in [1] and is redrawn in Fig. 3 for the sake of clarity and completeness. In this figure, the italic numbers denote the labels of the distribution lines, and buses are identified by the number positioned inside of them.

The experiments were divided into cases 1, 2, and 3. Case 1 allows the original real and reactive power demands values in [1] to vary within a tolerance of $8 \%$. Fig. 4(a and b) shows the ranges of each bus. Case 2 considers distinct perturbations to the load values on each bus. Fig. 5( $a$ and $b$ ) shows the ranges of the load values of each bus. Case 3 uses the demand curves obtained in [28] to describe seasonal variations in loads according to five profiles. These profiles were assigned to each load bus of the original test feeder in [1] according to the range of the value loads. Uncertainties were incorporated by assigning specified ranges to each bus by considering the minimal and peak values ( $\equiv$ nominal values of the original test feeder) of each profile (Fig. 6(a and b)). It is noteworthy that the uncertainties in cases 2 and 3 are more significant than in case 1 and that they are related to considerable oscillations in the load demand.

Computational experiments were performed using Matlab version 7.10.0.499 (R2010a) and the INTLAB toolbox [24], running on a i3 Core CPU with $2.13 \mathrm{GHz}$ and $4 \mathrm{~GB}$ of RAM memory, to evaluate the simulations involving interval analysis. The following evolutionary algorithm parameters were held fixed: population size (60), maximum number of generations (30), and probabilities of crossover (0.98) and mutation (0.05).

\subsection{Case 1}

In Table 1, the first and second data columns present the results obtained in [15] and from the MOEA-DFR, respectively, for an uncertainty-free 70-busbar feeder problem. The two sets of results are in agreement, which validates the MOEA-DFR in the uncertainty-free case. The initial configuration was assumed to be the same as that used in [15]. It is important to note that even though the proposed formulation in [15] was multi-objective, the fitness assignment was performed by a weighted-sum of objectives, and the authors provided only a single solution rather than a Pareto frontier. The MOEA-DFR generates a frontier, and we have shown that the Huang solution is contained on the achieved frontier. The last two data columns in Table 1 show the ranges of each objective of the Huang solution and one solution pertaining to the best frontier achieved using IMOEA-DFR, respectively, when uncertainties are included in the model. In this case, the two solutions are non-dominated by each other under Definition 6 (Section 4). Therefore, given the uncertainties in case 1 , both solutions can be properly adopted in real-world environments. In contrast to other methods, the IMOEA-DFR accounts for uncertainties in the modeling, and the robustness of the solutions is thus guaranteed. It is valuable to mention that the MOEA-DFR attained the Huang solution for ten distinct executions.

\subsection{Case 2}

Assuming the same uncertainty for all loads is naive because each bus generally has a distinct demand profile. Hence, in case 2 , we assumed different levels of uncertainty for each load values. Fig. 5 presents the power demand range at each load bus. In case 2 , we worked with three scenarios: (i) the original scenario described in [15], (ii) a perturbed scenario in which the uncertainties lead the load values to the boundary opposite that they were in original scenario when considering the power demand range in Fig. 5, and (iii) the interval scenario represented by the ranges at each bus in Fig. 5. Scenarios (i) and (ii) were solved by MOEA-DFR, while scenario (iii) was solved with the IMOEA-DFR. The frontiers obtained in the experiments in case 2 were built from ten distinct executions of the MOEA-DFR and IMOEA-DFR, whose parameters were adjusted to default values, as previously explained. The final frontier of each scenario was built by joining all non-dominated solutions belonging to the optimal frontier in each execution. Table 2 presents the distributions of the solutions found in each simulated scenario according to the number of switching operations. The MOEA-DFR found frontiers $\mathrm{Fr}_{o}$ and $\mathrm{Fr}_{p}$, which have 40 and 65 feasible solutions, respectively, to the simulated 70-busbar system in original and perturbed scenarios. The intersection between $\mathrm{Fr}_{O}$ and $\mathrm{Fr}_{P}$ contained only the initial solution because the fourth objective of the formulation requires that this solution remains on the final frontier of both sets (see Section 3). Thus, we conclude that all solutions in $\mathrm{Fr}_{O}$ are unfeasible or suboptimal configurations in the perturbed scenario. To obtain configurations that are suitable in all combinations of the original and perturbed scenarios, we use IMOEA-DFR to solve the interval scenario, which considers sufficiently large uncertainty margins to encompass the original and perturbed possibilities. IMOEA-DFR found frontier $\mathrm{Fr}_{I}$ with 75 feasible solutions, of which fourteen solutions were shared with $\mathrm{Fr}_{O}$ and four solutions with $\mathrm{Fr}_{P}$. As expected, only the initial solution exists at the intersection between frontiers $\mathrm{Fr}_{O}, \mathrm{Fr}_{P}$ and $\mathrm{Fr}_{I}$. 
It is worthwhile to note that the Huang solution is not included in $\operatorname{Fr}_{P}$ and $\mathrm{Fr}_{I}$. Thus, it is an unfeasible or suboptimal solution under the worst case uncertainties in case 2 . Table 3 shows that it is suboptimal in the perturbed scenario, consequently it still remains feasible. However, we cannot guarantee suboptimality or unfeasibility for other combinations of uncertainties within the range described in Fig. 5. In contrast, the solutions provided by IMOEA-DFR are assured to be robust in the worst cases of all uncertainties envisaged in the model.

\subsection{Case 3}

In this case, we assume that the nominal loads specified in [15] behave as described in [28] by using 24-h demand curves. Each of the six classes was delimited by intervals for classification purposes. Load values below $5 \mathrm{kVA}$ are classified as urban lighting. The rural, urban residential, commercial, and light industrial load intervals are 5-15 kVA, 15-100 kVA, 100-400 kVA, and 400$800 \mathrm{kVA}$, respectively. Apparent powers larger than $800 \mathrm{kVA}$ are associated with heavy industrial loads. The IMOEA-DRF solution, illustrated in Table 3, is compared to the Huang and original configurations (switches 70, 71, 72, 73 and 74 open) at seven instants in time. Fig. 7 clearly shows that the IMOEA-DRF solution outperforms the other solutions.

Table 4 provides more details about the performance of the different configurations. The cumulative power losses for each configuration are 524.2452 (original configuration - 100\%), 291.6970 (Huang - 55.64\%), and 273.6005 (solution proposed by IMOEADRF - 52.19\%). Although the number of switching operations is included in our multi-objective formulation as in many other published works, a more distant configuration can be better in aggregate by preventing more maneuvers in subsequent situations. In this example, the IMOEA-DFR configuration is achieved using eight switching maneuvers, but there is no need to perform other reconfigurations due to load variations throughout the day. Given that switching maneuvers are avoided by supervisory centers unless they are critical due to the risks involved in such operations, stable configurations are desired.

Using the computational environment and the algorithm parameters previously described, IMOEA-DFR has spent an averaged runtime of 2294.02, 2271.67, and $2223.83 \mathrm{~s}$ for cases I, II, and III, respectively. Those averages were calculated for ten runs of the algorithm performed in each case. The overall mean runtime is around $2263 \mathrm{~s}$ (75 s/generation), almost 65 times greater than the time spent by the conventional MOEA-DFR. As expected, the interval analysis has caused an increase on the computing burden due to extra calculus made to an interval instead of a single value. However, the benefit comes from the identification of robust configuration proper to be employed in longer periods outperforming other configurations achieved by means of the conventional approach, as verified in Fig. 7.

Unlike mono-objective problems, solving multi-objective problems consists in finding a set of solutions. Indeed, the quality of such solutions is evaluated by metrics which reveal some key factors: set size, closeness of the solution set to the Pareto frontier, and distribution pattern over the n-dimensional space. In this work, the performance of the solutions is evidenced by the hypervolume (HV) and Inverse Generational Distance (IGD) metrics. HV calculates the volume of the region, in the objective space, which is covered by the non-dominated solutions. Table 5 presents the HV values obtained by IMOEA-DFR. Regarding HV metric, the algorithm obtained better results for cases I and III. Table 6 presents the mean values of IGD metric for each run of those three cases. This metric was taken in relation to the estimated Pareto frontier. Smaller IGDs correspond to better convergence of the evolutionary algorithm. As HV, the IGD metric indicated that IMOEA-DFR obtained better results for cases I and III. Hence, the algorithm found more difficulty in case 2 (a scenario with high levels of uncertainty).

In summary, all of the experimental cases revealed that the non-robust solutions found by the MOEA-DFR perform similarly to the robust solutions achieved by the IMOEA-DFR whenever light perturbations are considered. However, the IMOEA-DFR outperformed the non-interval approach under severe load variations.

\section{Conclusions}

In this paper, the feeder reconfiguration problem in radial distribution networks was analyzed. We demonstrated that configurations obtained from uncertainty-free models may not perform as well as expected in the presence of uncertainties. Hence, load uncertainties must be taken into account to obtain a more realistic model that yields robust solutions, and the IMOEA-DFR is proposed to solve DFR in the occurrence of such uncertainties. Our results demonstrated that a configuration requiring more initial switching operations can be cumulatively better over long periods of time, as the aggregate number of switching maneuvers is minimized over time by preventing further reconfigurations necessary to handle load variations. In summary, our proposed method properly solves the DFR problem in uncertain power demand environment. The IMOEA-DFR successfully finds robust and reliable configurations in terms of safety, full compliance with power demand and constraints, which are each fundamental in practical situations.

\section{Acknowledgements}

This work has been supported in part by the Brazilian agencies CAPES and CNPq.

\section{References}

[1] Baran ME, Wu FF. Optimal capacitor placement on radial distribution systems. EEE Trans Power Del 1989;4(1):725-34.

[2] Baran ME, Wu FF. Network reconfiguration in distribution systems for loss reduction and load balancing. IEEE Trans Power Del 1989;4(2):1401-7.

[3] Barbosa CHNR, Caminhas WM, Vasconcelos JA. Adaptive technique to solve multi-objective feeder reconfiguration problem in real time context. In: Proc of the sixth int conf evolutionary multi-criterion opt, EMO 2011, Springer, Ouro Preto, Brazil; 2011. p. 418-32.

[4] Borges CT, Martins VF. Multistage expansion planning for active distribution networks under demand and distributed generation uncertainties. Int J Electr Power Energy Syst 2012;36:107-16.

[5] Broadwater RP, Shaalam HE, Fabrycky WJ, Lee RE. Decision evaluation with interval mathematics: a power distribution system case study. IEEE Trans Power Del 1994;9(1):59-67.

[6] Chaturvedi A, Prasad K, Ranjan R. Use of interval arithmetic to incorporate the uncertainty of load demand for radial distribution system analysis. IEEE Trans Power Del 2006:21(2):1019-21.

[7] Chen T, Lin E, Yang N, Hsieh T. Multi-objective optimization for upgrading primary feeders with distributed generators from normally closed loop to mesh arrangement. Int J Electr Power Energy Syst 2013:45:413-9.

[8] Chiang HD, Jumeau RJ. Optimal network reconfigurations in distribution systems: Part 2 - Solution algorithms and numerical results. IEEE Trans Power Del 1990;5(3):1568-74.

[9] Chiang HD, Jumeau RJ. Optimal network reconfigurations in distribution systems: Part 1 - A new formulation and a solution methodology. IEEE Trans Power Del 1990;5(4):1902-9.

[10] Civanlar S, Grainger JJ, Yin H, Lee SSH. Distribution feeder reconfiguration for loss reduction. IEEE Trans Power Del 1988;3(3):1217-23.

[11] Das B. Radial distribution system power flow using interval arithmetic. Int J Electr Power Energy Syst 2002;24(10):827-36.

[12] Das B. Consideration of input parameter uncertainties in load flow solution of three-phase unbalanced radial distribution system. IEEE Trans Power Syst 2006;21(3):1088-95.

[13] Deb K, Pratap A, Agarwal S, Meyarivan T. A fast and elitist multiobjective genetic algorithm: NSGA-II. IEEE Trans Evol Comp 2002;6(2):182-97.

[14] Deb K, Gupta H. Introducing robustness in multi-objective optimization. Evol Comp 2006;14(4):463-94.

[15] Huang YC. Enhanced genetic algorithm-based fuzzy multi-objective approach to distribution network reconfiguration. IEE Proc Gen Transm Distrib 2002;149(5):615-20. 
[16] Lopez E, Opazo H, Garcia L, Bastard P. Online reconfiguration considering variability demand: applications to real networks. IEEE Trans Power Syst 2004;19(1):549-53.

[17] Moore RE, Kearfoot RB, Cloud MJ. Introduction to interval analysis. Philadelphia (PA): SIAM; 2009.

[18] Niknam T. An efficient multi-objective HBMO algorithm for distribution feeder reconfiguration. Expert Syst Appl 2011;38:2878-87.

[19] Niknam T, Azadfarsani E, Jabbari M. A new hybrid evolutionary algorithm based on new fuzzy adaptive PSO and NM algorithms for distribution feeder reconfiguration. Energy Convers Manage 2012;54:7-16.

[20] Niknam T, Fard AK, Baziar A. Multi-objective stochastic distribution feeder reconfiguration problem considering hydrogen and thermal energy production by fuel cell power plants. Energy 2012;42:563-73.

[21] Niknam T, Kavousifard A, Aghaei J. Scenario-based multiobjective distribution feeder reconfiguration considering wind power using adaptive modified particle swarm optimisation. IET Renew Power Gener 2012;6(4):236-47.

[22] Niknam T, Kavousifard A, Tabatabaei S, Aghaei J. Optimal operation management of fuel cell/wind/photovoltaic power sources connected to distribution networks. J Power Sources 2011;196:8881-96.

[23] Niknam T, Zare M, Aghaei J, Farsani EA. A new hybrid evolutionary optimization algorithm for distribution feeder reconfiguration. Appl Artif Intell 2011:25:951-71.

[24] Rump SM. INTLAB: INTerval LABoratory. In: Developments in reliable computing. Dordrecht: Kluwer Academic Publishers; 1999. p. 77-104.

[25] Sahoo NC, Ranjan R, Prasad K, Chaturvedi A. A fuzzy-tuned genetic algorithm for optimal reconfigurations of radial distribution network. Eur Trans Electr Power 2007;17(2):97-111.
[26] Schmidt HP, Ida N, Kagan N, Guaraldo JC. Fast reconfiguration of distribution systems considering loss minimization. IEEE Trans Power Syst 2005;20(3):1311-9.

[27] Shariatkhah M, Haghifam M, Salehi J, Moser A. Duration based reconfiguration of electric distribution networks using dynamic programming and harmony search algorithm. Int J Electr Power Energy Syst 2012;41:1-10.

[28] Shenkman AL. Energy loss computation by using statistical techniques. IEEE Trans Power Del 1990;5(1):254-8.

[29] Shirmohammadi D, Hong HW. Reconfiguration of electric distribution networks for resistive line losses reduction. IEEE Trans Power Del 1989;4(2):1402-98.

30] Soares GL, Guimarães FG, Maia CA, Vasconcelos JA, Jaulin L. Interval robust multi-objective evolutionary algorithm. In: IEEE congress on evolutionary comp, CEC2009, Trondheim, Norway; 2009. p. 1637-43.

31] Torres J, Guardado JL, Rivas-Dávalos F, Maximov S, Melgoza E. A genetic algorithm based on the edge window decoder technique to optimize power distribution systems reconfiguration. Int J Electr Power Energy Syst 2013;45:28-34.

32] Vaccaro A, Villacci D. Radial power flow tolerance analysis by interval constraint propagation. IEEE Trans Power Syst 2009;24(1):28-39.

[33] Vaccaro A, Canizares CA, Villacci D. An affine arithmetic-based methodology for reliable power flow analysis in the presence of data uncertainty. IEEE Trans Power Syst 2010;25(2):624-32.

[34] Wang Z, Alvarado FL. Interval arithmetic in power flow analysis. IEEE Trans Power Syst 1992;7(3):1341-9.

[35] Zhang P, Li W Wang S, Reliability-oriented distribution network reconfiguration considering uncertainties of data by interval analysis. Int J Electr Power Energy Syst 2012;34:138-44. 